\title{
Accuracy enhancement of biometric recognition using iterative weights optimization algorithm
}

\author{
Pallavi D. Deshpande ${ }^{1 *}$, Prachi Mukherji ${ }^{2}$ and Anil S. Tavildar ${ }^{2}$
}

\begin{abstract}
A new approach is proposed to quantitatively evaluate the binary detection performance of the biometric personal recognition systems. The importance of correlation between the overall detection performance and the area under the genuine acceptance rate (GAR) versus false acceptance rate (FAR) graph, commonly known as receiver operating characteristics (ROC) is recognized. Using the ROC curve, relation between $G A R_{\text {min }}$ and minimum recognition accuracy is derived, particularly for high security applications (HSA). Finally, effectiveness of any binary recognition system is predicted using three important parameters, namely $G A R_{\text {min, }}$ the time required for recognition and computational complexity of the computer processing system. The palm print (PP) modality is used to validate the theoretical basis. It is observed that by combining different useful feature-extraction techniques, it is possible to improve the system accuracy. An optimum algorithm to appropriately choose weights has been suggested, which iteratively enhances the system accuracy. This also improves the effectiveness of the system.
\end{abstract}

Keywords: False acceptance rate (FAR), Genuine acceptance rate (GAR), Receiver operating characteristics (ROC), Area under the curve (AUC), Score level summing, Optimal weights algorithm (OWA)

\section{Introduction}

Extensive work has been done in the area of biometric recognition using different traits such as face, finger print, iris, voice, different hand-based modalities, gait, etc. [1-4]. However, unimodal systems have their own advantages and limitations. They require only one sensor to acquire data. Therefore, the data acquisition becomes less expensive and more user-friendly. They also require limited signal processing time and less computational efforts. However, the performance of any unimodal system gets degraded due to several reasons like erroneous database, spoofing attacks, etc. [5-7]. The unimodal systems provide lower values of genuine acceptance rate (GAR) and low to middle range of accuracies [8-10]. The proposed work in this paper focuses on increasing this low value of minimum value of genuine acceptance rate $\left(G A R_{\min }\right)$ for unimodal systems, with a view to

\footnotetext{
* Correspondence: deshpallavi2011@gmail.com

'Department of E\&TC Engineering, Vishwakarma Institute of Information Technology, SPPU, Pune, India

Full list of author information is available at the end of the article
}

boost their accuracies. This is done using mathematical modeling of signal detection performance and derivation of accuracy in terms of GARmin for high security applications (HSA). In this paper, efforts are made for enhancing the accuracy of palm print (PP)-based recognition using different feature extraction techniques, such as use of different discrete wavelet transform (DWT) coefficients and combinations of different DWT coefficients. Compared to other traits, PP features have certain distinct advantages. PP modality provides reasonably good recognition accuracy. PP authentication ensures advantages such as very stable line features, low intrusiveness, and requires low resolution imaging. As the palm area is much larger, more distinctive features can be captured. [11-13].

The $\mathrm{GAR}_{\text {min }}$ decides the detection performance of the system. The paper clearly brings out the correlation between the system accuracy with area under the curve (AUC) of its "GAR versus FAR characteristics," commonly known as receiver operating characteristics (ROC) of the system. In order to validate theoretical concepts proposed, PP modality is used. For PP modality, various feature 
extraction techniques are used to increase AUC of its ROC characteristics. Further, an appropriate iterative optimal weights algorithm (OWA) is suggested to further enhance the accuracy of the system. Finally, effectiveness of any binary recognition system is computed in terms of GARmin, recognition time, and computational complexity.

The paper is organized as follows: after brief introduction, Section 2 outlines the work done by other researchers in this field. Section 3 is devoted for mathematical background for binary signal detection and definition of effectiveness of the system. Section 4 elaborates the proposed methodology for the biometric image data acquisition, feature extraction, and feature mapping. Section 5 includes the system effectiveness calculations, analysis of the recognition performance, and the discussions on results, followed by conclusions.

\section{Related work}

Researchers have used different biometric traits for personal recognition purposes. As our focus is mainly on enhancing accuracy of unimodal hand-based systems, we have briefly reviewed research efforts, which reflect accuracies obtained for specific hand-based modalities.

E. Wong et al. (2008) [14] and M. Dale et al. (2009) [15] have worked on PP-based biometric recognition. They have reported accuracy levels of $94.84 \%$ and $97 \%$ respectively. Zhenhua Guo et al. (2011) [16] have used (2D) PCA for PP feature extraction. They have obtained an accuracy of $97.43 \%$. Goh Kahong Michael et al. (2012) [17] have described a contactless hand-based biometric system. They have extracted features using directional coding techniques, and normalized hamming distance has been used to find similarity between two feature sets. For individual modality, they have obtained GAR of 0.89 for hand geometry, 0.95 for PP, 0.75 for knuckle finger print, and 0.96 for finger vein modality. Kuang-Shyr Klu et al. (2013) [18] have used directional filter bank for palm vein modality in their work. They have quoted an accuracy of 99\%. Sibi Sasidharan et al. (2015) [19] have presented a paper in which they have given analysis of various methods and algorithms that identify the vein patterns for authentication purpose. They have concluded that palm vein recognition using neural network to be quite efficient and accurate. Raghavendra et al. (2015) [20] have explored the idea of PP recognition using a sparse representation of features obtained from Bank of Binarized Statistical Image Features (BSIF). Bank of BSIF that comprises of 56 different Bank of BSIF filters whose responses on the given PP modality image is processed independently and classified using sparse representation classifier (SRC). In the given PP sample, they obtained response on each of the Bank of BSIF filter and then they obtained the corresponding comparison score using SRC. Finally, they have selected the best comparison score that corresponds to the minimum value of the residual error. S. Khellat-Kihel et al. (2016) [21] have mentioned GAR values of 0.927, 0.846, 0.547 for finger knuckle print, finger vein, and fingerprint modalities respectively. Kunal Kumar et al. (2016) [5] have presented the strengths and weakness of selected biometric mechanisms and recommend novel solutions to include in multimodal biometric systems to improve on the current biometric drawbacks. Gopala et al. (2016) [6] have proposed fusion of PP, palm-phalanges print (PPP), and dorsal hand vein (DHV) in their paper. They have obtained GAR of $94 \%$ for PP, $98.2 \%$ for PPP, $92 \%$ for DHV, and GAR of $99.6 \%$ for score level fusion of PP-PPP-DHV. They have implemented score level fusion with conventional operators like Yager's ordered weighted averaging (OWA) operator and $t$ norm fusion operator. Shital Baghel et al. (2017) [7] have mentioned the drawbacks of unimodal systems compared to multimodal systems. Gopal Chaudhary et al. (2017) [8] have developed system using biometric trait, PPP. They have used different feature extraction techniques such as histograms of oriented gradients, Gaussian membership function (GMF) feature, mean value, and average absolute deviation method. Anchal Bansal et al. (2018) [9] have presented a review of different features of fingerprint recognition systems. The invariant and discriminatory information present in the fingerprint images are captured using fingerprint ridges known as minutiae. They have compared pattern recognition-based approach with wavelet-based approaches. Rupali L. et al. (2014) [22] have proposed the score level fusion of face and fingerprint modalities. Minutiae matching and Gabor filter techniques have been used for fingerprint recognition and principal component analysis for face recognition. After performing score normalization, score level fusion was done using simple sum rule. They have quoted an overall accuracy of $97.5 \%$ with FAR and FRR of $1.3 \%$ and $4.01 \%$ respectively. Assuming occurrence of equiprobable hypotheses, this represents a percentage accuracy of approx. 97.235\%. Furthermore, FAR level mentioned makes this approach not at all suitable for HSA, which is the prime focus of the present paper. Kamer Vishi et al. (2017) [23] have combined the normalized scores obtained from finger-veins and fingerprints modalities using different score level fusion techniques. They have implemented four score fusion approaches namely minimum score, maximum score, simple sum, and user specific weighting. They have considered using user specific weighting because they found that some biometric traits cannot be reliably obtained from some people. Hence, they have given a lower weight to a fingerprint score and a higher weight to a finger-vein score to reduce the probability of a false 
rejection. However, it appears that the weight assignment is rather on intuitive basis and not on any mathematical logic. As mentioned earlier, focus of the proposed work in this paper is mainly on enhancing accuracy of unimodal system so that it should be useful for HSA or very, very low FAR applications. Further, it may be observed that among various hand-based modalities, PP modality gives reasonably good accuracy. Therefore, focus of the proposed work is to explore the techniques, which may be useful for further improving the recognition accuracy of the PP modality based on logical, sound mathematical approach. The proposed unimodal system gives enhanced accuracy of $99.25 \%$ with very low FAR level of 0.0001 which is substantially greater than present day multimodal systems quoted above. Before explaining image capture, processing, and feature extraction, we introduce the mathematical basis for binary signal detection, with a view to understand the relationship between important parameters like $\mathrm{GAR}_{\min }, \mathrm{ROC}$, accuracy, etc. in the following section.

\section{Mathematical preliminaries}

3.1 Mathematical background for binary signal detection

The problem of binary detection is formulated and analyzed in various signal detection and estimation books $[24,25]$. The simplest binary communication system is shown in Fig. 1. A typical simple case consists of a single observation of the received signal corrupted by additive noise. The input signal is assumed to be in the binary form with two distinct values " $m_{\mathrm{o}}$ " and " $m_{1}$," corresponding to two binary hypotheses, " $H_{0}$ " and " $H_{1}$ " respectively. The received signal " $r$ " can be expressed as,

$$
r / H_{i}=m / H_{i}+\text { noise }(\mathrm{i}=0 \text { or } 1)
$$

In the above Eq. (1), symbol " $r / H_{i}$ " represents received signal " $\mathrm{r}$ " assuming hypothesis " $H_{\mathrm{i}}$ " is true and symbol " $m / H_{i}$ " represents mean value of the received signal assuming hypothesis " $H_{\mathrm{i}}$ " is true. The last term "noise" represents undesirable degradations due to fine dust, illumination effects, blurring effects, etc. In this paper, it is further assumed that overall effect of all degradations due to central limit theorem of statistics leads to zero mean Gaussian distribution with variance " $\sigma^{2}$." Further, the probability density function (PDF) for the observed signal can be expressed as,

$$
P(r / H i) \otimes G\left(m_{i}, \sigma^{2}\right)
$$

In Eq. (2), symbol “ " means equality in PDF and "G" represents standard Gaussian distribution. For the simplest example of binary hypothesis testing considered, the generalized likelihood ratio test (GLRT) has been derived as [24, 25],

$$
\operatorname{GLRT}=\frac{\mathrm{P}\left(\mathrm{r} \mid \mathrm{H}_{1}\right)}{\mathrm{P}\left(\mathrm{r} \mid \mathrm{H}_{0}\right)} \stackrel{\substack{\mathrm{H}_{1} \\ \underset{\mathrm{H}_{0}}{>}}}{<}
$$

In Eq. (3), " $\gamma$ " represents decision threshold. For the simple case of single observation, the above equation leads to,

$$
r \stackrel{\substack{H_{1} \\ \underset{H_{0}}{>}}}{>}
$$

In Eq. (4), the term " $\gamma$ " is given by Eq. (5).

$$
\gamma=\frac{\sigma^{2}}{m_{1}} \ln (\eta)+m_{1} / 2
$$

In Eq. (3), signal value for the hypothesis " $H_{0}$ " has been assumed to be zero (i.e., $m_{0}=0$ ) for simplicity and in Eq. (5), " $\eta$ " represents ratio of occurrence of a priori probabilities of two hypotheses $H_{0}$ and $H_{1}$. For equiprobable hypotheses, " $\eta=1$ " and decision threshold becomes " $\gamma=m_{1} / 2$ " which is expected.

The GAR can now be expressed as,

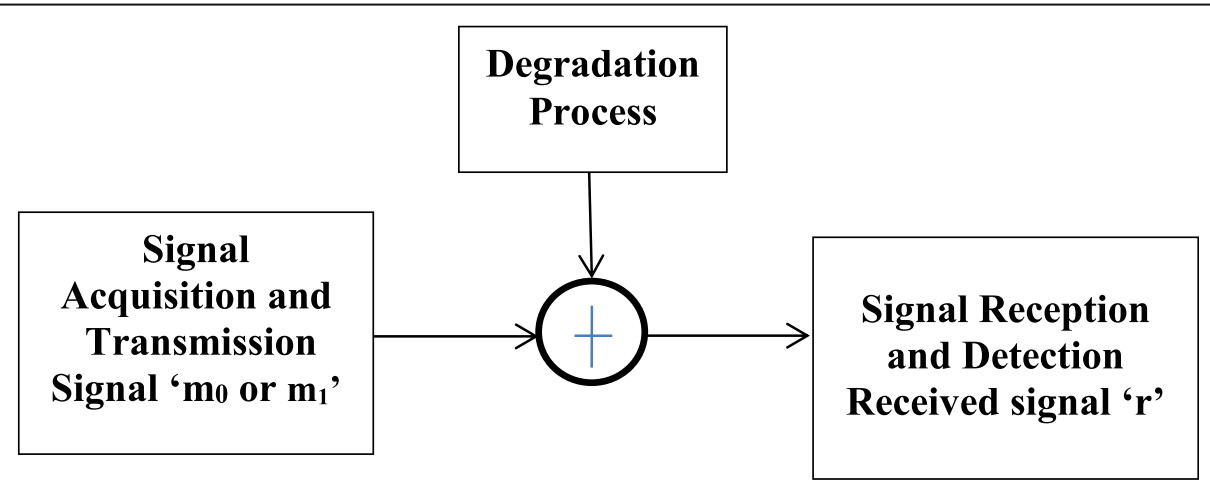

Fig. 1 The simplest binary communication system 


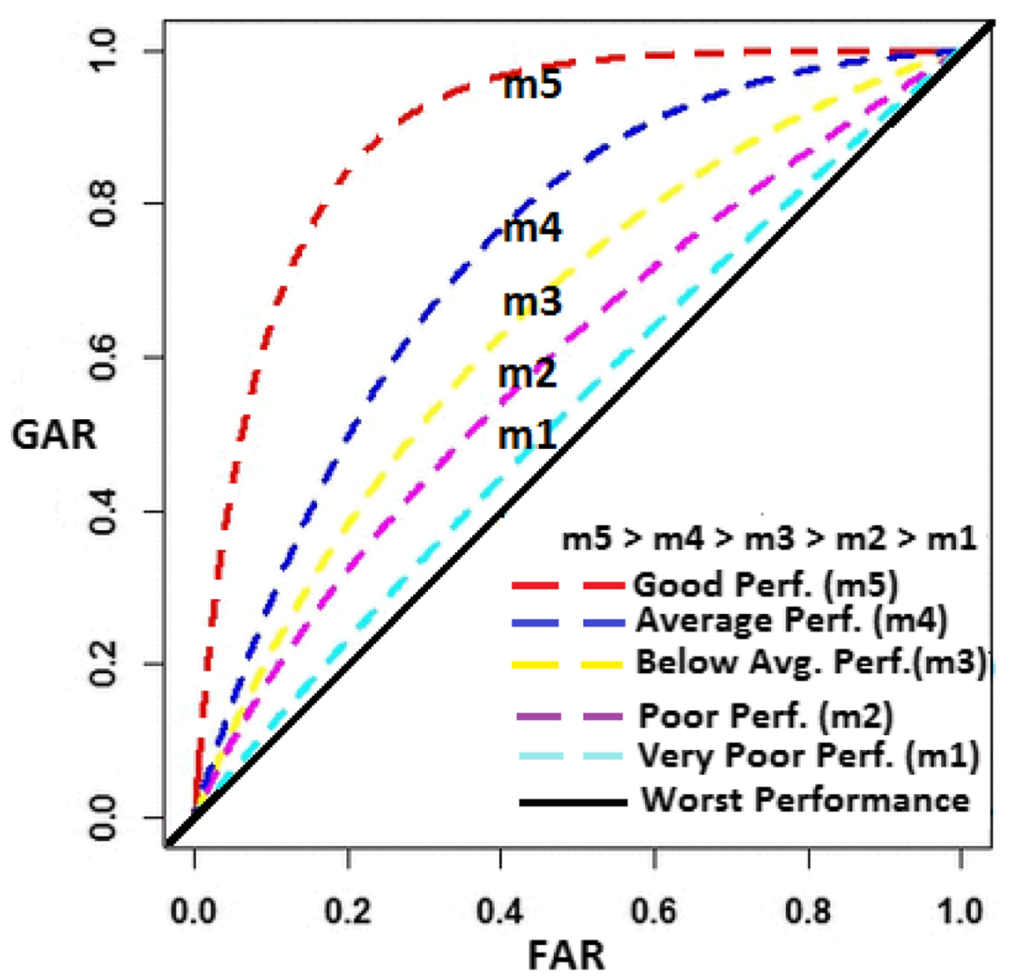

Fig. 2 ROC curves for different signal values

$$
\mathrm{GAR}=\int_{\gamma}^{\infty} \mathrm{P}\left(\mathrm{r} \mid \mathrm{H}_{1}\right) \mathrm{dr}
$$

Similarly, FAR can be expressed as,

$$
\mathrm{FAR}=\int_{\gamma}^{\infty} \mathrm{P}\left(\mathrm{r} \mid \mathrm{H}_{0}\right) \mathrm{dr}
$$

In biometric terms GAR and FAR are known as "True Positive" and "False Positive" respectively. The ROC curve represents plot of "GAR versus FAR" as value of decision threshold " $\gamma$ " is varied from very low value to very large value. As " $\gamma$ " threshold increases, both GAR and FAR get reduced. In this case, we follow the test suggested by Neyman-Pearson (NP) [24], in which decision threshold is pre decided by level of maximum FAR permissible in the application. In this method, GAR is maximized for the stipulated value of FAR. In high security applications, value of FAR $\leq 0.0001$ (maximum of one among 10,000 samples may be falsely acceptable). Hence, we set our threshold based on equation no. (7), with FAR $=0.0001$.

Typical ROC characteristics for the simplest case are shown in the Fig. 2, where typical signal values, $m_{5}>$ $m_{4}>m_{3}>m_{2}>m_{1}$ are used and " $m_{0}$ " is assumed to be zero. The ideal characteristics would be $G A R=1$ and

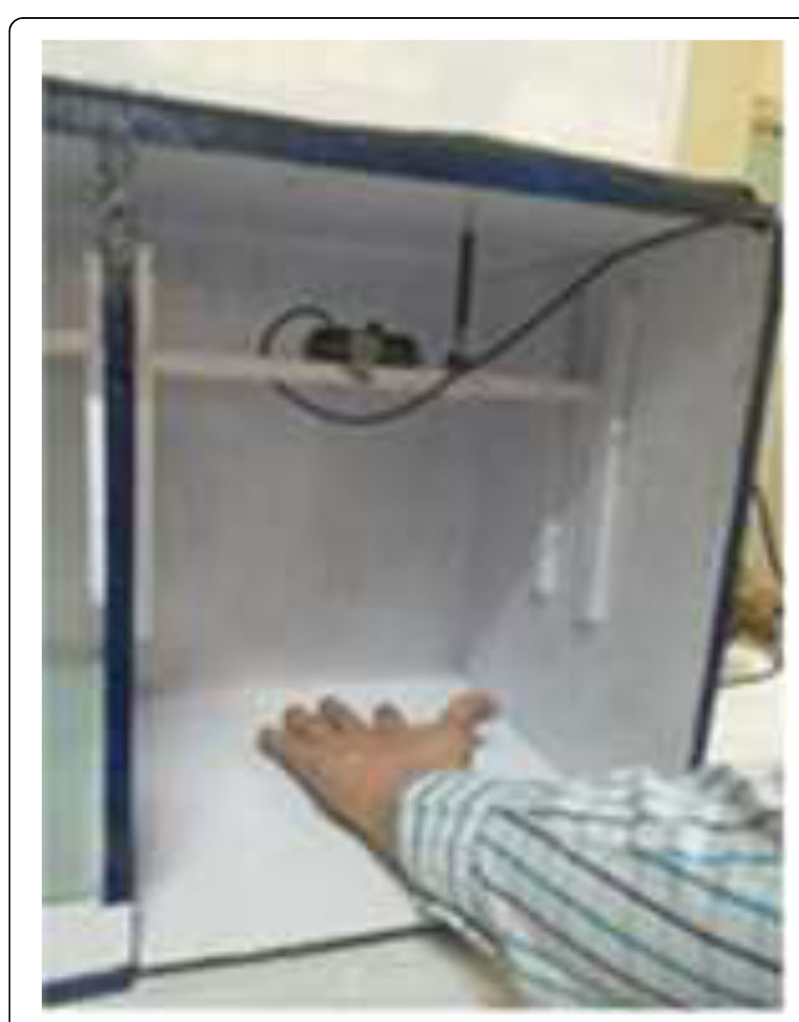

Fig. 3 Data acquisition system 
$F A R=0$ and the worst scenario, GAR $=$ FAR which is diagonal solid line marked as "Worst Performance" in Fig. 2. It is observed that as the signal value increases, the ROC curve shifts toward the ideal curve and the detection performance improves [24].

The error in the binary system can be defined as,

$E_{\mathrm{r}}=\left[P_{0} \times(\right.$ false acceptance rate $)+P_{1} \times($ genuine rejection rate $\left.)\right]$

In Eq. (8), genuine rejection rate (GRR) represents false negative for biometric system. Further, $P_{0}$ and $P_{1}$ represent a priori probabilities of hypotheses $H_{0}$ and $H_{1}$ respectively. Assuming two hypotheses to be equally likely, i.e., $P_{0}=P_{1}=1 / 2$, the error situation can be expressed as,

$$
E_{\mathrm{r}}=(\mathrm{FAR}+\mathrm{GRR}) / 2
$$

The detection accuracy can be expressed as,

$$
\text { Accuracy }=(1-\mathrm{Er})
$$

For the simple equiprobable binary hypothesis, Eq. (10) leads to,

$$
\text { Accuracy }=\left(1-\frac{(F A R+(1-G A R))}{2}\right)
$$

Further, for high security applications, where FAR is very small compared to GAR, above Eq. (11) approximates to,

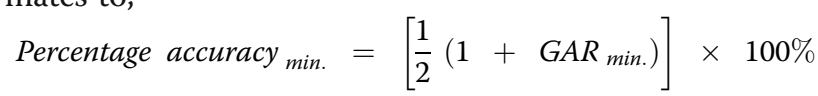

It is readily seen from the $R O C$ that $G A R_{\min }$ is the value of GAR, at which the ROC curves depart from $\mathrm{FAR}=0$ (i.e., $Y$-axis) tangentially. Further, as a value of AUC $(0.5<$ AUC $<1.0)$ increases, the value of $G A R_{\text {min }}$ also increases, which leads to accuracy enhancement. For the worst scenario, $\mathrm{GAR}_{\min }=0$. For ideal scenario, $\mathrm{GAR}_{\text {min }}$ approaches to 1 . Thus the accuracy level increases from 50 to $100 \%$. One more commonly used method in binary recognition is equal error rate (EER) method, i.e., $F A R=$ GRR. In this case, for equiprobable hypotheses, the accuracy in \% units can be expressed as,

$$
\begin{aligned}
& \text { Percentage accuracy }=(1-\text { GRR }) \times 100 \\
& \text { Percentage accuracy }=(\text { GAR }) \times 100
\end{aligned}
$$

However, in this case, GAR has to be determined at the EER point. In this paper, as our main focus is on high security applications, we use only NP test explained above and evaluate the percentage accuracy using Eq. (12).

\subsection{System effectiveness}

The term "system effectiveness $\epsilon$ " represents the quantitative measure of effectiveness of biometric recognition system, which can be defined as follows,

$$
\text { System effectiveness }(\varepsilon)=\left(\frac{\text { GAR }_{\text {min. }} \times t_{\text {max. }}}{t}\right) \times C c
$$

In Eq. (15), " $t_{\max }$ " represents maximum permissible time as per system requirements stipulated and " $t$ "
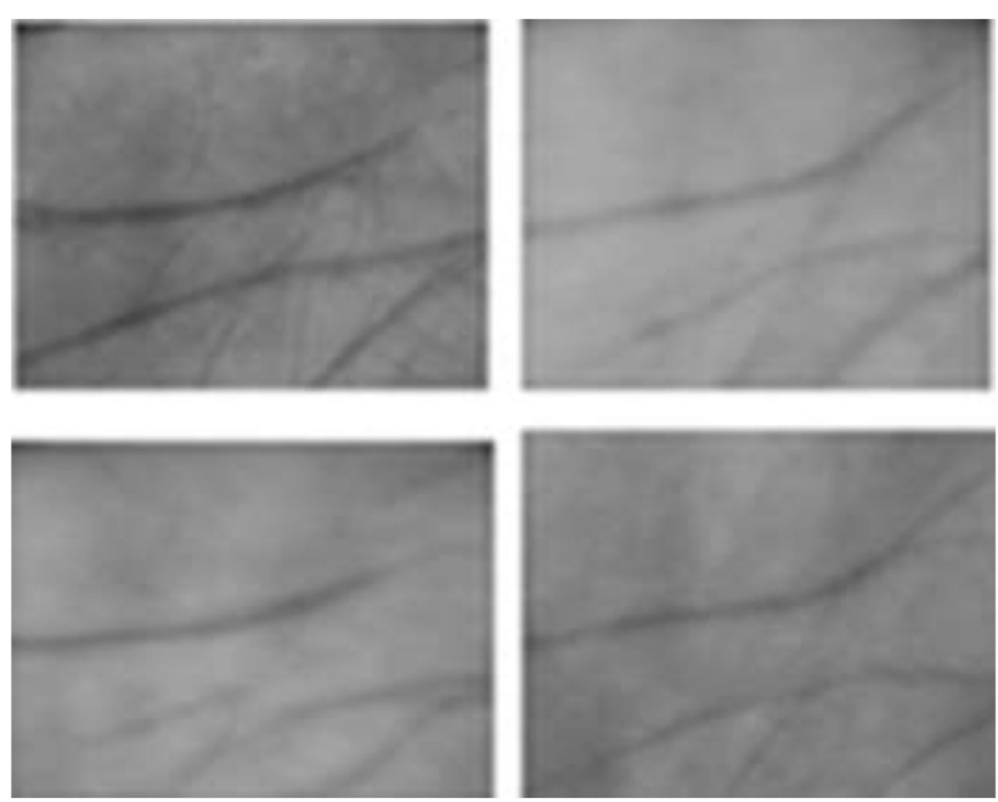

Fig. 4 Enhanced palm prints $\mathrm{ROI}$ images 
represents actual time required for recognition process. Further, " $C_{C}$ " represents the hardware complexity of the computer configuration used. Effectiveness of the system increases with increase in "GAR min" which enhances the recognition accuracy and with reduction in the actual recognition time $t$. The system effectiveness also increases with increase in complexity of the computer configuration used. The higher the value of system effectiveness, the better will be the biometric system.

With above analytical background and definitions, we now consider the image acquisition, feature extraction techniques, and features mapping techniques used for the biometric recognition system.

\section{Image data acquisition, feature extractions, and feature mapping techniques}

4.1 Image capture process

The data acquisition system (DAS) has been designed, developed, and fabricated. Regarding this DAS and the required image pre-processing, we have explained in detail in our paper published during the Conference on Advances in Signal Processing (CASP), 2016 [11]. DAS to extract PP modality is shown in Fig. 3. Webcam with medium resolution $640 \times 480$ has been used to acquire images and database of 150 users has been created. In this case, all 150 users were asked to wash/clean their hands before image capture process. This ensures that the database was reasonably error free data and only noise during capture can lead to the degradation of images. For every user, ten images of PP modality have been captured. Extraction of PP ROI has been discussed in detail in the paper published during International Conference on Electronics and Communication Systems (ICECS), 2014 [12]. The enhanced ROIs for palm print images are shown in Fig. 4. The biometric feature extraction is discussed in the following subsection.

\subsection{Techniques for palm print feature extraction}

Two techniques namely Harris Corner Detector (HCD) and DWT are used for feature extraction. These techniques are briefly discussed below:

\subsubsection{Feature extraction using $H C D$}

HCD algorithm was used to extract palm print feature vector [26]. Algorithm is stepwise explained in the following.

Step 1: Compute $X$ and $Y$ derivatives of image by convolving image " $"$ " with Prewitt operator.

$$
I_{x}=G_{\sigma}^{x} * I, I_{y}=G_{\sigma}^{y} * I
$$

In Eq. $16, G_{\sigma}^{x}=$ Prewitt vertical edge operator and $G_{\sigma}^{y}$ $=$ Prewitt horizontal edge operator
Step 2: Compute products of derivatives at every pixel.

$$
I_{\mathrm{x}}^{2}=I_{\mathrm{x}} \cdot I_{\mathrm{x}}, I_{\mathrm{y}}^{2}=I_{\mathrm{y}} \cdot I_{\mathrm{y}}, I_{\mathrm{x}} I_{\mathrm{y}}=I_{\mathrm{x}} \cdot I_{\mathrm{y}}
$$

Step 3: Compute the sums of the products of derivatives at each pixel.

$$
\begin{aligned}
I_{\mathrm{x} 2} & =G_{\sigma^{\prime}} * I_{\mathrm{x}}^{2}, I_{\mathrm{y} 2}=G_{\sigma^{\prime}} * I_{\mathrm{y}}^{2}, I_{\mathrm{xy}} \\
& =G_{\sigma^{\prime}} * I_{\mathrm{x}} I_{\mathrm{y}}
\end{aligned}
$$

In Eq. (18), $G_{\sigma^{\prime}}=$ Gaussian filter

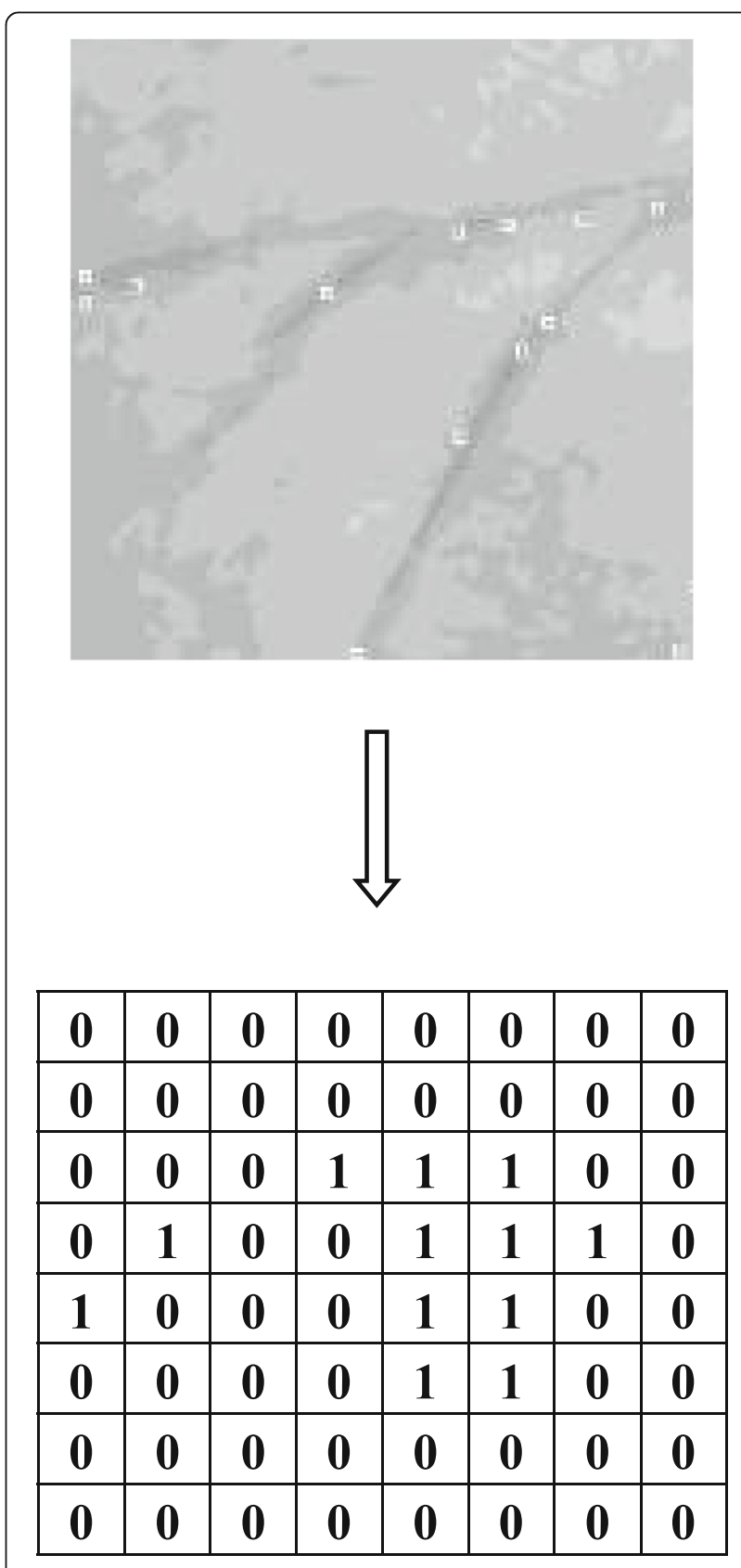

Fig. 5 Palm print with corners and its binary matrix 
Step 4: Define at each pixel $(x, y)$ the matrix.

$$
M=\left[\begin{array}{ll}
I_{\mathrm{x} 2}(x, y) & I_{\mathrm{xy}}(x, y) \\
I_{\mathrm{xy}}(x, y) & I_{\mathrm{y} 2}(x, y)
\end{array}\right]
$$

Step 5: Compute the "response" of the detector at each pixel.

$$
R=\operatorname{Det}(M)-K(\operatorname{Trace}(M))^{2}
$$

In Eq. (20), " $K$ " is a constant, which lies in between the range 0.04 to $0.07[12,26]$. All pixels that have scores greater than a certain threshold value " $R$ " have been marked as the "Corner points or Tracking points" on the ROI of palm print image. We have taken the size of this ROI 256 by 256 pixels. Number of corner points lies in between 12 and 16 depending upon the value of " $K$ " $[12,26]$. These corner points have been mapped from ROI of 256 by 256 pixels into a binary matrix of size $8 \times 8$, as shown in Fig. 5. Thus, PP feature vector has been created. HCD has few limitations. HCD consumes substantial time to detect the corners. Further, as HCD uses spatial domain processing, the accuracies may be adversely affected due to intensity artifacts, rotation effects, degradation effects, etc.

\subsubsection{Palm print feature extraction using DWT}

The methodology for extraction of DWT features has been explained in detail in Pallavi Deshpande et al. [11]. As indicated therein, two vectors of size $25 \times 54$, one for the approximate coefficients and the other for horizontal coefficients for each of the image, are stored in the database. In this paper, a novel algorithm for optimally choosing the weights of the approximate and horizontal coefficients to create a final feature vector of the PP image is proposed to enhance the accuracy of the system. Further, empirical-based technique and optimal-based "optimum weights algorithm" are used to decide the weights of approximate and horizontal coefficients in the combined feature vectors to boost the accuracy.

\subsection{Feature matching}

\subsubsection{Methodology for mapping HCD features}

Feature vector, i.e., binary matrix of new user (size 8 by 8 ), is compared with every stored feature matrix (each of size 8 by 8 ) in the training database. Logical "AND" operation between this new user's matrix is done with each and every feature vector/matrix present in the database. For genuine user, maximum number of 1's will be obtained through this logical ANDING operation in the output matrix. Finally, summation of all 1's in the output matrix will give the overall score. The maximum score gives best match as shown in Fig. 6 [12].

\begin{tabular}{|l|l|l|l|l|l|l|l|}
\hline 0 & 0 & 0 & 0 & 0 & 0 & 0 & 0 \\
\hline 0 & 0 & 0 & 0 & 0 & 0 & 0 & 0 \\
\hline 0 & 0 & 0 & 1 & 1 & 1 & 0 & 0 \\
\hline 0 & 1 & 0 & 0 & 1 & 1 & 1 & 0 \\
\hline 1 & 0 & 0 & 0 & 1 & 1 & 0 & 0 \\
\hline 0 & 0 & 0 & 0 & 1 & 1 & 0 & 0 \\
\hline 0 & 0 & 0 & 0 & 0 & 0 & 0 & 0 \\
\hline 0 & 0 & 0 & 0 & 0 & 0 & 0 & 0 \\
\hline
\end{tabular}

\begin{tabular}{|l|l|l|l|l|l|l|l|}
\hline $\mathbf{0}$ & $\mathbf{0}$ & $\mathbf{0}$ & $\mathbf{0}$ & $\mathbf{0}$ & $\mathbf{0}$ & $\mathbf{0}$ & $\mathbf{0}$ \\
\hline $\mathbf{0}$ & $\mathbf{0}$ & $\mathbf{0}$ & $\mathbf{0}$ & $\mathbf{0}$ & $\mathbf{0}$ & $\mathbf{0}$ & $\mathbf{0}$ \\
\hline $\mathbf{0}$ & $\mathbf{0}$ & $\mathbf{0}$ & $\mathbf{1}$ & $\mathbf{1}$ & $\mathbf{1}$ & $\mathbf{0}$ & $\mathbf{0}$ \\
\hline $\mathbf{0}$ & $\mathbf{1}$ & $\mathbf{0}$ & $\mathbf{0}$ & $\mathbf{1}$ & $\mathbf{1}$ & $\mathbf{1}$ & $\mathbf{0}$ \\
\hline $\mathbf{1}$ & $\mathbf{0}$ & $\mathbf{0}$ & $\mathbf{0}$ & $\mathbf{1}$ & $\mathbf{1}$ & $\mathbf{0}$ & $\mathbf{0}$ \\
\hline $\mathbf{0}$ & $\mathbf{0}$ & $\mathbf{0}$ & $\mathbf{0}$ & $\mathbf{1}$ & $\mathbf{1}$ & $\mathbf{0}$ & $\mathbf{0}$ \\
\hline $\mathbf{0}$ & $\mathbf{0}$ & $\mathbf{0}$ & $\mathbf{0}$ & $\mathbf{0}$ & $\mathbf{0}$ & $\mathbf{0}$ & $\mathbf{0}$ \\
\hline $\mathbf{0}$ & $\mathbf{0}$ & $\mathbf{0}$ & $\mathbf{0}$ & $\mathbf{0}$ & $\mathbf{0}$ & $\mathbf{0}$ & $\mathbf{0}$ \\
\hline
\end{tabular}

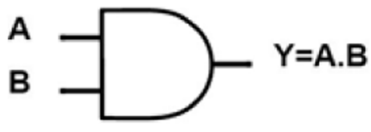

\begin{tabular}{|l|l|l|l|l|l|l|l|}
\hline $\mathbf{0}$ & $\mathbf{0}$ & $\mathbf{0}$ & $\mathbf{0}$ & $\mathbf{0}$ & $\mathbf{0}$ & $\mathbf{0}$ & $\mathbf{0}$ \\
\hline $\mathbf{0}$ & $\mathbf{0}$ & $\mathbf{0}$ & $\mathbf{0}$ & $\mathbf{0}$ & $\mathbf{0}$ & $\mathbf{0}$ & $\mathbf{0}$ \\
\hline $\mathbf{0}$ & $\mathbf{0}$ & $\mathbf{0}$ & $\mathbf{1}$ & $\mathbf{1}$ & $\mathbf{1}$ & $\mathbf{0}$ & $\mathbf{0}$ \\
\hline $\mathbf{0}$ & $\mathbf{1}$ & $\mathbf{0}$ & $\mathbf{0}$ & $\mathbf{1}$ & $\mathbf{1}$ & $\mathbf{1}$ & $\mathbf{0}$ \\
\hline $\mathbf{1}$ & $\mathbf{0}$ & $\mathbf{0}$ & $\mathbf{0}$ & $\mathbf{1}$ & $\mathbf{1}$ & $\mathbf{0}$ & $\mathbf{0}$ \\
\hline $\mathbf{0}$ & $\mathbf{0}$ & $\mathbf{0}$ & $\mathbf{0}$ & $\mathbf{1}$ & $\mathbf{1}$ & $\mathbf{0}$ & $\mathbf{0}$ \\
\hline $\mathbf{0}$ & $\mathbf{0}$ & $\mathbf{0}$ & $\mathbf{0}$ & $\mathbf{0}$ & $\mathbf{0}$ & $\mathbf{0}$ & $\mathbf{0}$ \\
\hline $\mathbf{0}$ & $\mathbf{0}$ & $\mathbf{0}$ & $\mathbf{0}$ & $\mathbf{0}$ & $\mathbf{0}$ & $\mathbf{0}$ & $\mathbf{0}$ \\
\hline
\end{tabular}

Fig. 6 Logical AND operation performed on two binary matrices 


\section{a Receiver Operating Characteristics}

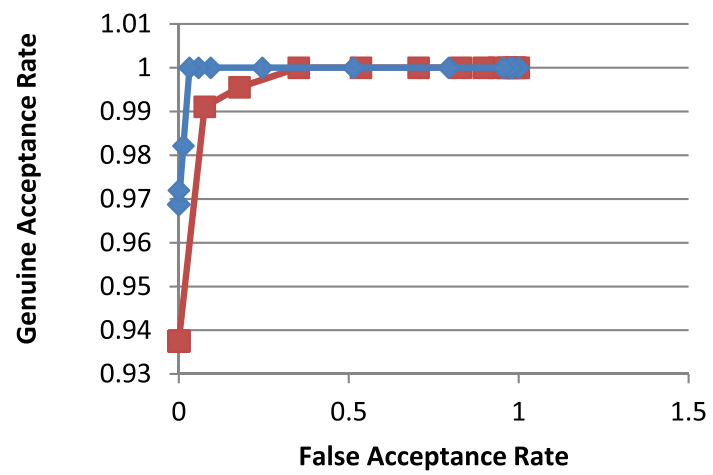

- Palm Print with

Horizontal

Coefficients

Palm Print with

Approximate

Coefficients

False Acceptance Rate

\section{b Receiver Operating Characteristics}

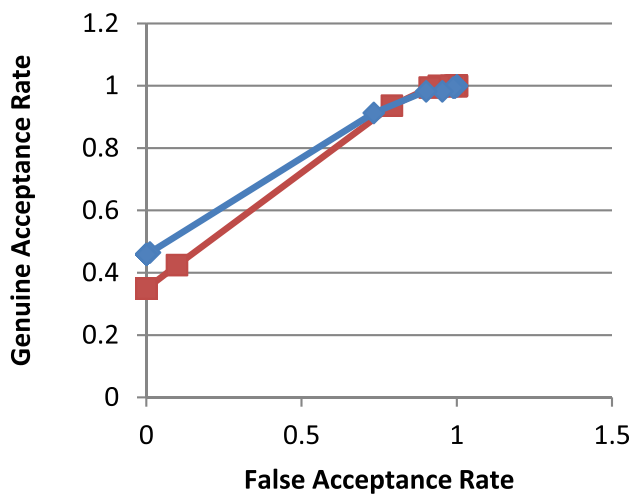

-Palm Print with

Vertical Coefficients

$\sim$ Palm Print with

Diagonal Coefficients

Fig. 7 a ROC curves plotted using approximate and horizontal wavelet coefficients for PP modality. b ROC curves plotted using vertical and diagonal wavelet coefficients for PP modality

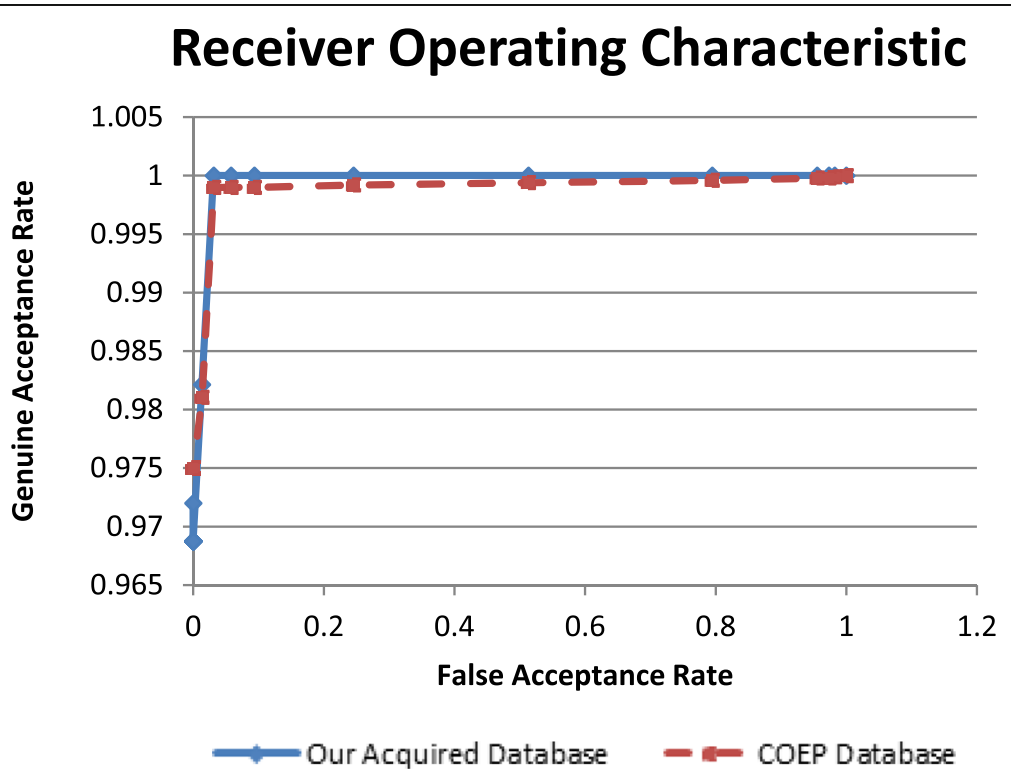

Fig. 8 ROC of palm print modality for both the databases using approximate coefficients only 


\section{Receiver Operating Characteristics}

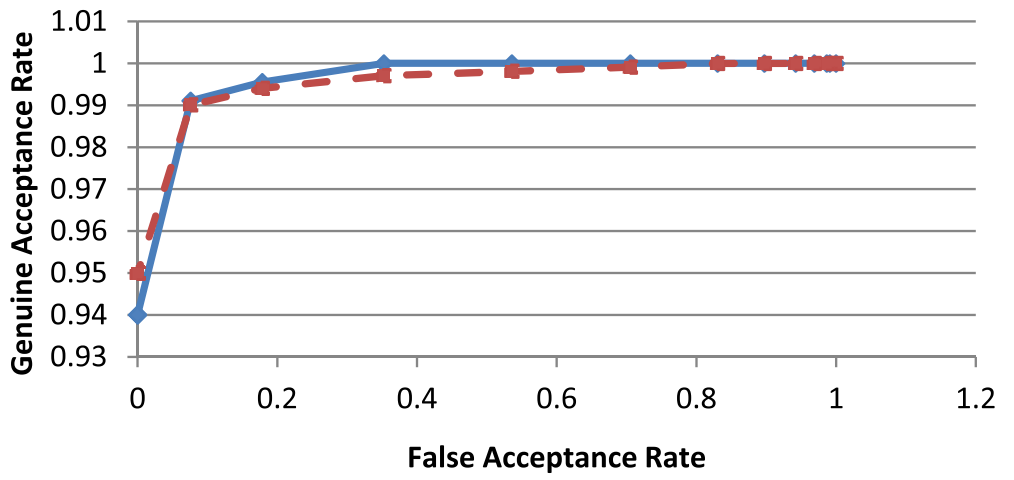

- Our Acquired Database - - COEP D atabase

Fig. 9 ROC of palm print modality for both the databases using horizontal coefficients only

\subsubsection{Methodology for mapping DWT features}

In this case, the Euclidean distance (E.D.) is computed between the new user's DWT feature vector of size $25 \times$ 54 and every stored DWT feature vector (of size $25 \times 54$ ) in the database using Eq. (21),

$$
\text { E.D. }=\sqrt{\sum_{i=1}^{L}\left(x_{\mathrm{i}}-y_{\mathrm{i}}\right)^{2}}
$$

In this case, " $x_{1}, x_{2} \ldots x_{n}$ " represent coefficients from feature vector of new user and " $y_{1}, y_{2} \ldots y_{\mathrm{n}}$ " represent coefficients from feature vector of stored palm print and " $L$ " represents size of feature vector which is of size $25 \times$ 54. The minimum Euclidean distance gives the best match.

\subsection{Methodology for accuracy prediction}

Different techniques are used to create feature vectors for the proposed PP based biometric system. Their accuracy estimation methods are given below.

\subsubsection{HCD technique-based biometric recognition system}

For the HCD features, considering the correct assessment of GAR using the acquired database, $\mathrm{GAR}_{\min }$ has been estimated as 0.9536 . This is based on the entire mapping process carried out within the database, where out of 220 images, the exact match was found for 210 images. For rest of the ten images, there was mismatch, thus giving $\mathrm{GAR}_{\min }$. 0.9536 . Based on this, accuracy for high security applications works out to $97.68 \%$ using Eq. (12). Even though the above accuracy appears to be good, due to need for pixel by pixel mapping and totally spatial domain signal processing, as mentioned earlier, the HCD would be more likely to degrade the accuracy performance further. The DWT, on the other hand, has some distinct advantages.

DWT technique uses joint time-frequency domain approach, hence pixel by pixel comparison is not required. Further, the degradations due to rotation, size, and brightness effects are much less severe for DWT [13, 27]. Typical ROC curves for PP modality using DWT technique are plotted for acquired error free database of 150 users and are shown in Fig. 7a, b. All four types of DWT coefficients (i.e., approximate, horizontal, vertical, and diagonal) are extracted and ROCs are plotted for all four types of DWT coefficients. After comparing ROC characteristics of all four types of DWT coefficients, it can be readily seen that a ROC for approximate coefficients is very close to the ideal ROC curve (AUC lies in between 0.95 and 1.0). ROCs for vertical and diagonal coefficients are far away from the ideal curve (in fact they are nearer to the worst scenario, i.e., AUC equals to 0.50). The ROC curve for horizontal coefficient lies in

Table 1 Comparison using three feature extraction techniques

\begin{tabular}{|c|c|c|c|c|c|}
\hline \multirow{2}{*}{$\begin{array}{l}\text { Sr. } \\
\text { No. }\end{array}$} & \multirow[t]{2}{*}{ Method used } & \multicolumn{2}{|c|}{ Acquired database } & \multicolumn{2}{|c|}{ COEP database } \\
\hline & & $\overline{\mathrm{GAR}_{\min }}$ & $\overline{\text { Accuracy }}$ & $\overline{\mathrm{GAR}_{\min }}$ & $\overline{\text { Accuracy }}$ \\
\hline 1 & Harris Corner Detector & 0.9536 & $97.68 \%$ & - & - \\
\hline 2 & DWT with approximate coefficients & 0.972 & $98.60 \%$ & 0.975 & $98.75 \%$ \\
\hline 3 & DWT with horizontal coefficients & 0.940 & $97.00 \%$ & 0.950 & $97.50 \%$ \\
\hline
\end{tabular}


between the two extremes. Therefore, it is proposed to use "only approximate coefficients" or "only horizontal coefficients" or "the combination of approximate plus horizontal coefficients" for the formation of the feature vectors. Analysis and further enhancing accuracy using approximate and horizontal DWT coefficients is discussed in the following subsection.

\subsubsection{Feature vectors using "only approximate" DWT coefficients-based biometric recognition system}

Using feature vectors based on only approximate DWT coefficients, as per methodology discussed in Section 4.2.2, ROCs for our acquired PP database of 150 users and the standard PP database provided by College of Engineering, Pune (COEP) are shown in Fig. 8. As explained earlier, tangential departure of the ROC from $\mathrm{FAR}=0$ (i.e., $Y$-axis) gives the values for $\mathrm{GAR}_{\min }$ and AUC values.

Following performance level can be determined using the ROC curve plotted for our own acquired database.

- $\mathrm{AUC}_{\mathrm{APP}}=0.975$

- $\mathrm{GAR}_{\min }=0.972$

- Accuracy $_{\min }=98.60 \%$ using Eq. (12)

For benchmarking purposes, the standard publically available COEP database is used. (http://www.coep. org.in/resources/coeppalmprintdatabase). This database consists of eight different images of single user having resolution of $1600 \times 1200$ pixels per image. The database consists of total 1344 images pertaining to 168 users. Figure 8 also includes ROC of the palm print modality using COEP database. Following performance level can be determined using the ROC curve.

- $\mathrm{AUC}_{\mathrm{APP}}=0.98$

- $\mathrm{GAR}_{\min }=0.975$

- Accuracy $_{\min }=98.75 \%$

\subsubsection{Feature vectors using "only horizontal" DWT coefficients-based biometric recognition system}

Using identical approach as explained in Section 4.2.2 ROCs are plotted using only horizontal DWT coefficients. The ROC curves for both the databases are shown in Fig. 9. The parameters namely $\mathrm{AUC}_{\mathrm{HPP}}$ and $\mathrm{GAR}_{\min }$ are determined and accuracy is calculated using Eq. (12) for acquired database as shown below.

- $\mathrm{AUC}_{\mathrm{HPP}}=0.9211$

- $\mathrm{GAR}_{\min }=0.94$

- Accuracy $_{\min }=97.00 \%$

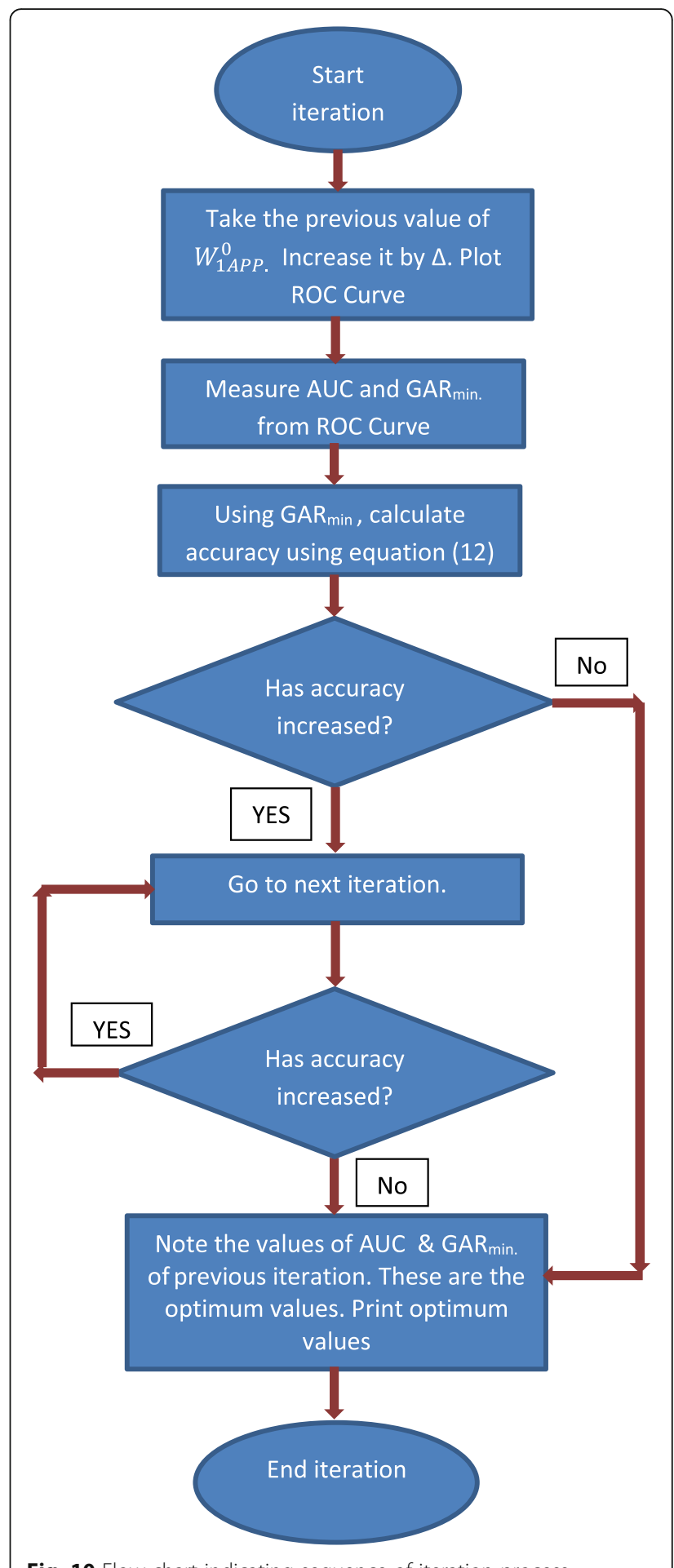

Fig. 10 Flow chart indicating sequence of iteration process

Similarly, Fig. 9 also includes ROC of the palm print modality for COEP database. Following performance level is determined using the ROC curve.

- $\mathrm{AUC}_{\mathrm{HPP}}=0.94$

- $\mathrm{GAR}_{\text {min }}=0.95$ 
Table 2 Summary of parameters at different iterations for our acquired database

\begin{tabular}{|c|c|c|c|c|c|}
\hline Sr. No. of iteration (i) & $W_{1 A P P}^{i}$ & $W_{2 H P P}^{i}$ & $\mathrm{GAR}_{\min }$ & $\%$ of minimum accuracy achievable & Performance \\
\hline 0 & 0.513227513 & 0.486772487 & 0.9800 & 99.00 & Good \\
\hline 1 & 0.563227513 & 0.438172487 & 0.9810 & 99.05 & Good \\
\hline 2 & 0.613227513 & 0.389572487 & 0.9810 & 99.05 & Good \\
\hline 3 & 0.663227513 & 0.340822487 & 0.9810 & 99.05 & Good \\
\hline 4 & 0.713227513 & 0.292072487 & 0.9820 & 99.10 & Better \\
\hline 5 & 0.763227513 & 0.243247487 & 0.9820 & 99.10 & Better \\
\hline 6 & 0.813227513 & 0.191322487 & 0.985 & 99.25 & Best \\
\hline 7 & 0.863227513 & 0.144422487 & 0.9820 & 99.10 & Better \\
\hline 8 & 0.913227513 & 0.09522386 & 0.9810 & 99.05 & Good \\
\hline
\end{tabular}

- Accuracy $_{\min }=97.50 \%$

Table 1 shows GAR $_{\text {min }}$ and accuracy values using three feature extraction techniques for PP recognition. Comparing the accuracies for DWT features for our database and COEP standard database, it is seen that our algorithm gives comparable performance levels for both databases. This indicates that the algorithm proposed can very well be used irrespective of database. As accuracies predicted for our own database (which is error free database) and COEP database are almost same, it also suggests that COEP database is also fairly clean and error free database.

The accuracies indicated in Table 1 are good. However, in order to further boost accuracies, "Empirical method" or "OWA" can be used. These methods are discussed in the following subsections.

\subsection{Performance prediction using combination of feature vectors}

Two different methods namely "OWA" and "Empirical method" are discussed below to combine approximate and horizontal DWT coefficients to create new feature vectors in order to boost accuracy of the system.

\subsubsection{Performance prediction using analytical approach}

Suppose there are " $n$ " coefficients be combined, the generalized formulation to decide the weights of coefficients of the system can be expressed as

$$
W_{n}=\propto \times A U C_{n} \text { with, } \sum_{n=1}^{N} W_{n}=1
$$

In Eq. (22), each of $W_{\mathrm{n}}\left(0<W_{\mathrm{n}} \leq 1\right)$ represents weight for the $n$th coefficient with AUC for ROC curve denoted by $\mathrm{AUC}_{\mathrm{n}}\left(0.5<\mathrm{AUC}_{\mathrm{n}} \leq 1\right)$. In our case, we consider only two coefficients, approximate and horizontal. Therefore, $N=2$. For deciding optimum values of weights $W_{1 \text { APP }}$ and $W_{2 \mathrm{HPB}}$ areas under two ROCs of acquired database (shown in Figs. 7 and 8 respectively) are to be considered.
The proportionality constant " $\alpha$ " can now be determined using

$$
W_{1 \mathrm{APP}}+W_{2 \mathrm{HPP}}=1
$$

Above Eq. (23) leads to the equation,

$$
W_{n}=\frac{A U C_{n}}{\sum_{1}^{N} A U C_{n}}
$$

In the proposed system, considering $\mathrm{AUC}_{\mathrm{APP}}=0.975$, $\mathrm{AUC}_{\mathrm{HPP}}=0.9211$, we get $\alpha=0.5291005291$, giving values of $W_{1 A p p}^{0}=0.5132275132$ and $W_{2 H P P}^{0}=0.4867724868$.

We now commence our iteration process for the zeroth iteration $(i=0)$ with above values of $W_{1 A P P}^{0}$ and $W_{2 H P P}^{0}$ By using these weights, area under the revised ROC curve $\left(A U C^{0}\right.$ ) was 0.9720 and $G_{A R}$ min was measured as 0.9800 . This results in accuracy of $99.00 \%$ using Eq. (12). The performance can be further improved through series of iterations as illustrated below.

For this, it was observed that $\mathrm{AUC}_{\mathrm{APP}}>\mathrm{AUC}_{\mathrm{HPP}}$. Therefore, we go on increasing weight of approximate coefficients " $W_{1 A P P}^{0}$ " in small positive steps $(\Delta=0.05)$ in each iteration and weight of horizontal coefficients " $W_{2 H P P}^{0}$ " has to be decreased appropriately.

The general equations for iterations are as follows.

$$
W_{1 A P P}^{i+1}=W_{1 A P P}^{i}+\Delta
$$

and

Table 3 Results obtained for different combinations of weights

\begin{tabular}{lllll}
\hline Sr. No. & $E_{\text {SUMPP }}$ & \multicolumn{2}{l}{ Our acquired database } \\
\cline { 3 - 5 } & & GAR & Accuracy & Performance \\
\hline 1 & {$\left[\left(0.5 \times E_{\mathrm{APP}}\right)+\left(0.5 \times E_{\mathrm{HPP}}\right)\right]$} & 0.9810 & 99.05 & Good \\
2 & {$\left[\left(0.6 \times E_{\mathrm{APP}}\right)+\left(0.4 \times E_{\mathrm{HPP}}\right)\right]$} & 0.9810 & 99.05 & Good \\
3 & {$\left[\left(0.7 \times E_{\mathrm{APP}}\right)+\left(0.3 \times E_{\mathrm{HPP}}\right)\right]$} & 0.9820 & 99.10 & Better \\
4 & {$\left[\left(0.8 \times E_{\mathrm{APP}}\right)+\left(0.2 \times E_{\mathrm{HPP}}\right)\right]$} & 0.984 & 99.20 & Best \\
5 & {$\left[\left(0.85 \times E_{\mathrm{APP}}\right)+\left(0.15 \times E_{\mathrm{HPP}}\right)\right]$} & 0.9810 & 99.05 & Good \\
\hline
\end{tabular}


Table 4 Comparison between two configurations

\begin{tabular}{llll}
\hline Sr. No. & Parameters & Configuration 1 & Configuration 2 \\
& & Dell Inspiron N5050 & HP Pavilion 15 cc134tx \\
\hline 1 & Processor used & Intel I 2350M & Intel Core i7 \\
2 & Clock speed & $2.3 \mathrm{GHz}$ & $4.0 \mathrm{GHz}$ \\
3 & Processor generation & 2nd generation & 8th generation \\
\hline
\end{tabular}

$$
W_{2 H P P}^{i+1}=W_{2 H P P}^{i}-\Delta \times A U C^{i}
$$

In Eqs. (25) and (26), $W^{i+1}$ represents the weight assigned for $(i+1)$ iteration, where $i=1,2,3,4 \ldots .$. represents the iteration sequence number and $A U C^{i}$ represents area under the corresponding ROC curve after $i$ th iteration. After each iteration, two parameters namely AUC and the new value of $G_{A R}$ min with revised ROC are determined. Using the GARmin, the accuracy prediction is done using Eq. (12). It is observed that after every iteration, the accuracy goes on increasing. Iteration process is continued until accuracy reaches to maximum. After this, any further increase in $W_{1 \mathrm{APP}}$ leads to reduced value of $\mathrm{GAR}_{\min }$ which decreases the accuracy. We stop the iteration process at this point. Typical sequence of iterations is depicted in Fig. 10. The iteration process implemented is illustrated in the following.

4.5.1.1 First iteration Let us take $i=1$. Take the previous value of $W_{1 \mathrm{APP}}^{0}=0.5132275132$ and $\Delta=0.05$. Using Eq. (25), we get $W_{1 \mathrm{APP}}^{1}=$ equal to 0.563227513 . Using Eq. (26), we get $W_{2 \mathrm{HPP}}^{1}$ equal to 0.438172487 . Using these new weights, the new revised ROC was plotted and the area under this new ROC curve $\left(\mathrm{AUC}^{1}\right)$ was 0.9720 and $\mathrm{GAR}_{\min }$ was measured as 0.9810 , giving an accuracy of $99.05 \%$. To improve the accuracy further, we continued the same process in second iteration.

4.5.1.2 Second iteration To explore further improvement possible, the weight for approximate coefficients was further increased by $\Delta=0.05$ to $W_{1 \mathrm{APP}}^{2}=0.61322$ 7513. Following exactly identical procedure, as in the first iteration, the new weights are determined as,

$$
\begin{aligned}
W_{1 A P P}^{2} & =0.613227513 \text { and } W_{2 H P P}^{2} \\
& =0.389572487
\end{aligned}
$$

Using these new weights, the new ROC was plotted and the area under this new ROC curve $\left(\mathrm{AUC}^{2}\right)$ was 0.9750 and $\mathrm{GAR}_{\min }$ was measured as 0.9820 , giving an accuracy of $99.10 \%$ which is marginally higher than the accuracy obtained in the first iteration. This process has been continued further. Table 2 shows the summary of the number of iterations carried out.

From Table 2, it can be observed that values of weights for the 6th iteration yields the highest maximum accuracy. Beyond this, the accuracy decreases for the subsequent iterations. Hence, those values of weights are considered as optimal values. Hence, the optimum values of the weights after 6th iteration are as follows, $W_{1 A P P}^{6}=0.813227513$ and $W_{2 H P P}^{6}=0.191322487$.

Using these new weights, the new ROC was plotted and the area under this new ROC curve was 0.9776 and $\mathrm{GAR}_{\text {min }}$ works out to be 0.9885 , giving an accuracy of $99.25 \%$, which is the highest possible value of the accuracy of the system. Based on the above optimum weight algorithm, Sum rule is,

$E_{S U M}=\left(0.813227513 \times W_{A P P}\right)+\left(0.191322487 \times W_{H P P}\right)$

\subsubsection{Weights selection using empirical method}

In addition to analytical method, one can use simple empirical method to decide the weights. The simple method is described in the following. After feature extraction, score normalization is done using " $\mathrm{Z}$ score" normalization technique [4]. In order to increase the accuracy of the system, the horizontal and approximate normalized scores are combined with the following sum-

\begin{tabular}{|c|c|c|c|}
\hline \multirow{2}{*}{$\begin{array}{l}\text { Sr. } \\
\text { No. }\end{array}$} & \multirow{2}{*}{$\begin{array}{l}\text { Acquired database } \\
\text { (approximate + horizontal } \\
\text { coefficients) }\end{array}$} & \multicolumn{2}{|c|}{ Mapping time required per image in seconds } \\
\hline & & $\begin{array}{l}\text { Configuration } 1 \\
\text { Dell Inspiron N5050 }\end{array}$ & $\begin{array}{l}\text { Configuration } 2 \\
\text { HP Pavilion } 15 \text { cc134tx }\end{array}$ \\
\hline 1 & 60 users $(60 \times 6=360$ hands $)$ & 1.88625 & 0.96312 \\
\hline 2 & 100 users $(100 \times 6=600$ hands $)$ & 2.99375 & 1.49687 \\
\hline 3 & 150 users $(150 \times 6=900$ hands $)$ & 4.115625 & 2.15781 \\
\hline
\end{tabular}
ming rule:

Table 5 Mapping time required using approximate plus horizontal coefficients 
Table 6 Mapping time required using only approximate coefficients

\begin{tabular}{|c|c|c|c|}
\hline \multirow{2}{*}{$\begin{array}{l}\text { Sr. } \\
\text { No. }\end{array}$} & \multirow{2}{*}{$\begin{array}{l}\text { Acquired database } \\
\text { (approximate coefficients) }\end{array}$} & \multicolumn{2}{|c|}{ Mapping time required per image in seconds } \\
\hline & & $\begin{array}{l}\text { Configuration } 1 \\
\text { Dell Inspiron N5050 }\end{array}$ & $\begin{array}{l}\text { Configuration } 2 \\
\text { HP Pavilion } 15 \text { cc134tx }\end{array}$ \\
\hline 1 & 60 users $(60 \times 6=360$ hands $)$ & 1.77797 & 0.88998 \\
\hline 2 & 100 users $(100 \times 6=600$ hands $)$ & 2.96562 & 1.48281 \\
\hline 3 & 150 users ( $150 \times 6=900$ hands $)$ & 3.948437 & 1.995618 \\
\hline
\end{tabular}

$$
E_{S U M}=\left[\left(W_{1 A P P} \times N S_{A P P}\right)+\left(W_{2 H P P} \times N S_{H P P}\right)\right]
$$

In Eq. 28, $W_{1 \mathrm{APP}}$ and $W_{2 \mathrm{HPP}}$ are the weights empirically chosen for normalized scores of approximate $\left(\mathrm{NS}_{\mathrm{APP}}\right)$ and horizontal $\left(\mathrm{NS}_{\mathrm{HPP}}\right)$ coefficients of PP modality respectively. Here, we start with equal weights $W_{1 \mathrm{APP}}=W_{2 \mathrm{HPP}}=0.5$. Comparing the two ROC curves, we know that $\mathrm{AUC}_{\mathrm{APP}}>\mathrm{AUC} \mathrm{C}_{\mathrm{HPP}}$. Therefore, weight of approximate coefficients is increased in the step of 0.1 and weight of horizontal coefficients is decreased accordingly. For each step, the new ROC is plotted and $\mathrm{GAR}_{\text {min }}$ is determined. The accuracy is determined using Eq. 12. The results of $G A R_{\text {min }}$ and accuracy for different empirical choices of $W_{1 \mathrm{APP}}$ and $W_{2 \mathrm{HPP}}$ are indicated in Table 3.

From Table 3, it can be observed that $\mathrm{GAR}_{\min }$ and accuracy values appear to be best for the following rule:

$$
E_{S U M}=\left[\left(0.8 \times N S_{A P P}\right)+\left(0.2 \times N S_{H P P}\right)\right]
$$

It is readily observed that the above weights based on empirical method are fairly closer to the weights suggested by "OWA" in Section 4.5.1.

\subsection{Evaluation of the system effectiveness}

Time required for extracting palm print features of a new users' hand image is observed to be approximately 3 se. After feature extraction, the average feature mapping time has also been recorded. While taking average, 20 iterations are done and average time of these twenty iterations is recorded. The average mapping time varies according to database size (i.e., 60, 100 or 150 users) and also depends upon the computational complexity in the hardware used. Comparison between the two configurations from hardware complexity view point is depicted in Table 4 below.
All the other parameters such as type of display, Cache memory, RAM/ROM sizes, etc. are not relevant for our application and therefore not considered for complexity estimation. Here, clock speeds for the two configurations are different. Cost for the two configurations would vary substantially and it would also depend upon local taxes, duties, etc. Therefore, the cost is not included in deciding $\mathrm{Cc}$ values. We determine the computational complexity "Cc" factor as follows:

$$
\begin{aligned}
\text { Cc for configuration } 1 & =\text { Dell Inspiron N5050 }=1.0 \\
\text { Cc for configuration } 2 & =\text { HP Pavilion } 15 \text { cc134tx } \\
& =4 \mathrm{GHz} / 2.2 \mathrm{GHz}=1.8181
\end{aligned}
$$

Tables 5 and 6 show the mapping time required for the two main feature extraction techniques namely, only approximate DWT coefficients and combined approximate plus horizontal DWT coefficients using above configurations. These two configurations were used for system effectiveness calculations as the two configurations were readily available with us at our research center. Form Tables 5 and 6 , it is seen that there is very small difference between mapping times for DWT methods using "only approximate coefficients" and using combined "approximate plus horizontal coefficients" for two different configurations. But the accuracy prediction results indicate that there is some definite improvement in accuracy when both "approximate and horizontal coefficients" are combined (refer serial no. 6 from Table no. 2).

It can be seen from Tables 5 and 6 that mapping time varies as per size of database. As expected, the mapping time increases if size of the database is increased from

\begin{tabular}{|c|c|c|c|c|}
\hline \multirow{2}{*}{$\begin{array}{l}\text { Sr. } \\
\text { No. }\end{array}$} & \multirow[t]{2}{*}{ Technique used } & \multirow[t]{2}{*}{$\mathrm{GAR}_{\min }$} & $t$ in seconds & \multirow{2}{*}{$\begin{array}{l}\text { System effectiveness } \\
\text { for } C_{C}=1.0\end{array}$} \\
\hline & & & $C_{C}=1.0$ & \\
\hline 1 & DWT with approximate coefficients & 0.972 & 2.96562 & 1.6387804 \\
\hline 2 & DWT with horizontal coefficients & 0.940 & 2.96560 & 1.5848394 \\
\hline 3 & DWT with approximate and horizontal coefficients & 0.985 & 2.99375 & 1.6450939 \\
\hline
\end{tabular}
60 to 100 and from 100 to 150 . Therefore, system effectiveness calculations will also change as per the size of the database. Just for illustration, a system effectiveness

Table 7 Summary of results for $C_{C}=1.0$ 
Table 8 Summary of results for $C_{C}=1.8181$

\begin{tabular}{|c|c|c|c|c|}
\hline \multirow{2}{*}{$\begin{array}{l}\text { Sr. } \\
\text { No. }\end{array}$} & \multirow[t]{2}{*}{ Technique used } & \multirow[t]{2}{*}{$\mathrm{GAR}_{\min }$} & $t$ in seconds & \multirow{2}{*}{$\begin{array}{l}\text { System effectiveness } \\
\text { for } C_{C}=1.8181\end{array}$} \\
\hline & & & $C_{C}=1.8181$ & \\
\hline 1 & DWT with approximate coefficients & 0.972 & 1.48281 & 5.9589 \\
\hline 2 & DWT with horizontal coefficients & 0.940 & 1.44232 & 5.9245 \\
\hline 3 & DWT with approximate and horizontal coefficients & 0.985 & 1.496875 & 5.9818 \\
\hline
\end{tabular}

calculation for the database of 100 users is shown in the next section.

\section{Results and discussion}

Based on the approach described in Section 4 above, the summarized results are presented in what follows. Further illustrative results for database of 100 users only are included.

\subsection{System effectiveness calculations for $N=100$}

Tables 7 and 8 summarize the results for system effectiveness for database of 100 users for the two configurations $C_{C}=1.0$ and $C_{C}=1.8181$ respectively. As discussed in Subsection 3.2, we use Eq. (15) for system effectiveness calculations.

It is clearly seen from Table 8 that when the "configuration 2" computing facility is used for combined "approximate and horizontal coefficients" technique with the use of weight optimization algorithm, the effectiveness increases to 5.9818. This is due to substantial higher clock frequency of configuration 2, as compared to configuration 1 .
5.2 Performance prediction using combination of feature vectors-based biometric recognition system

Figure 11 shows ROC of the palm print modality using both approximate and horizontal coefficients as per "optimal weights algorithm."

- $\mathrm{AUC}_{\mathrm{PP}}=0.9776$

- $\mathrm{GAR}_{\min }=0.985$

- Accuracy $_{\min }=99.25 \%$

Similar procedure was also carried out for COEP database for validation and benching marking of the optimal weights algorithm. Figure 11 also shows ROC of the palm print modality using both approximate and horizontal coefficients using optimum weights algorithm.

- $\mathrm{AUC}_{\mathrm{PP}}=0.97$

- $\mathrm{GAR}_{\min }=0.984$

- Accuracymin $_{\text {min }} 99.20 \%$

System gives an enhanced accuracy of $99.25 \%$ using this "optimal weights algorithm." From the above discussions, it can be seen that ROC and accuracy of PP modality using combined approximate and horizontal

\section{Receiver Operating Characteristics}

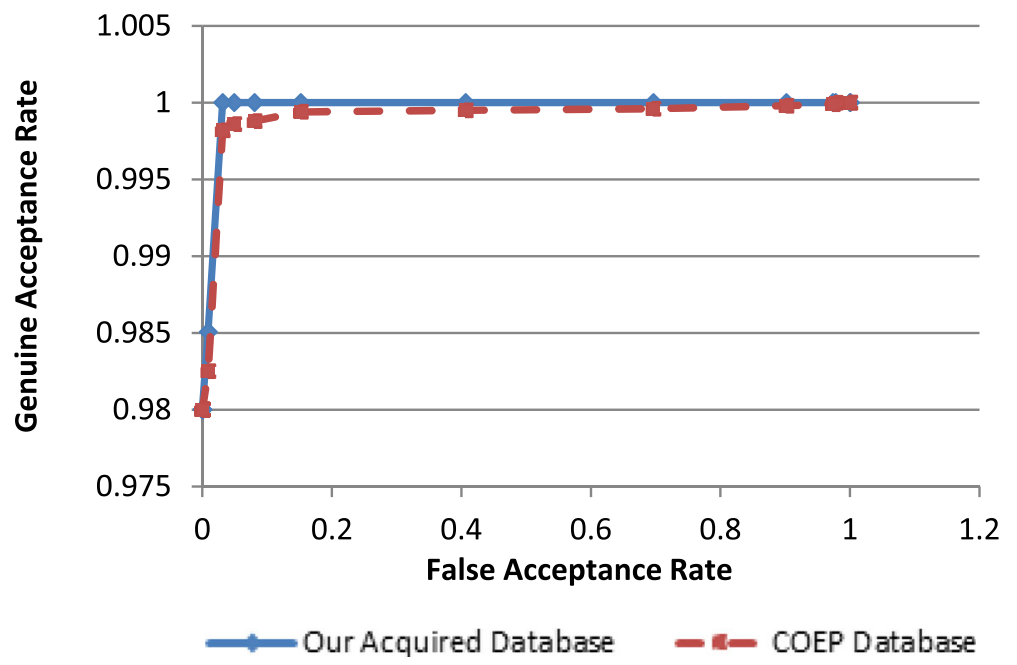

Fig. 11 ROC of palm print modality for both the databases using optimal weights algorithm for approximate and horizontal coefficients 
Table 9 Comparison of detection parameters among different methods

\begin{tabular}{llllll}
\hline Sr. & Method used & Acquired database & \multicolumn{2}{c}{ COEP database } \\
\cline { 3 - 4 } No. & & GAR $_{\min }$ & Accuracy & GAR & Accuracy \\
\hline 1 & Harris Corner Detector & 0.9536 & $97.68 \%$ & - & - \\
2 & Horizontal DWT coefficients only & 0.940 & $97.00 \%$ & 0.950 & $97.50 \%$ \\
3 & Approximate DWT coefficients only & 0.972 & $98.60 \%$ & 0.975 & $98.75 \%$ \\
4 & Combined approximate plus horizontal DWT coefficients using optimum weights algorithm. & 0.985 & $99.25 \%$ & 0.984 & $99.20 \%$ \\
\hline
\end{tabular}

coefficients are certainly better than ROC and accuracy of palm print modality using only approximate coefficients or using only horizontal coefficients. The summarized results using different techniques using our own database and also using a publically available COEP database are depicted in Table 9. It may be seen from Table 9 that our proposed "optimum weights algorithm" gives same enhanced accuracies using acquired database as well as COEP database. Further, we make use of only two techniques indicated at serial no. 3 and serial no.4 (refer to Table 9) as they provide best possible accuracies. It is proposed to use optimum weight algorithm with values of 0.81 for the approximate coefficients and 0.19 for horizontal coefficients, as it would provide best possible percentage accuracy.

\section{Conclusion and future scope}

The paper provides a mathematical basis for evaluating the accuracy of the biometric recognition system. This paper clearly brings out a strong correlation between the detection accuracy of the system with the area under the curve (AUC) of its ROC. It has been shown that combining various useful DWT features leads to enhancement in accuracy. Use of iterative optimal weights algorithm (OWA) is proposed to further improve the accuracy of the system. Testing, validation, and benchmarking of the algorithm are done using the acquired database, as well as with standard publically available COEP database. The proposed system gives enhanced accuracy of $99.25 \%$ with very low FAR level of 0.0001 . This represents fairly accurate and significantly user-friendly biometric system, suitable for higher security applications. Even though we have used the algorithm only for combining the two types of coefficients, the proposed optimization algorithm may be very effectively used for combining different normalized scores of any multimodal $(N>2)$ biometric recognition system, with a view to boost the accuracy and increase the effectiveness of multimodal systems. Further, one can also consider systematically extending the procedure to more number of modalities using the standard principles of mathematical induction.

\section{Abbreviations}

AUC: Area under the curve; DHV: Dorsal hand vein; DWT: Discrete wavelet transform; FAR: False acceptance rate; GAR: Genuine acceptance rate;
GAR min: Minimum value of genuine acceptance rate; HSA: High security applications; OWA: Optimal weights algorithm; PP: Palm print; PPP: Palmphalanges print; ROC: Receiver operating characteristics

\section{Symbols}

$\epsilon:$ System effectiveness; H: Ratio of occurrence of a priori probabilities of two

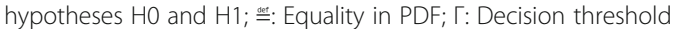

\section{Acknowledgements}

The authors would also like to specifically acknowledge the guidance/help extended by Prof. Dr. Yogesh H. Dandawate of V.I.IT., Pune, without which, this research work would not have been possible.

Funding

Not applicable.

\section{Availability of data and materials}

The datasets used and/or analyzed during the current study are available from the corresponding author on reasonable request.

\section{Authors' contributions}

All three authors made substantial contributions to conception and design, or acquisition of data, or analysis and interpretation of data. They have been involved in drafting the manuscript or revising it critically for important intellectual content. They have given final approval of the version to be published. Each author has participated sufficiently in the work to take public responsibility, for appropriate portions of the content and agreed to be accountable for all aspects of the work, in ensuring that questions related to the accuracy or integrity of any part of the work are appropriately investigated and resolved.

\section{Authors' information}

Pallavi D. Deshpande is pursuing her PhD at Vishwakarma Institute of Information Technology's research center, Pune, India. She has done her A.M.I.E.T.E., B.E. (Electronics) and M.E. (Digital Systems) from University of Pune and has been pursuing her Ph. D (Electronics and Telecommunications) from University of Pune. Her research interests include Signal and Image Processing, ANN, and Biometrics. She is Life Member of I.E.T.E., India.

Prachi Mukherji is working as a Professor and HOD in E\&TC Engineering department at MKSSS's Cummins College of Engineering for Women, Pune, India. She has obtained her B.E. (Electronics) in 1991 and her M. Tech (Digital Communication) in 1994. She has obtained her Ph.D. (Electronics and Telecommunications) in 2009 from University of Pune. She has a total of 25 years of teaching experience, out of which 10 years in research. Her research interests include Signal and Image Processing, Pattern Recognition and Communication Engineering. Till date, she has published 45 papers in the National and International Journals and conferences.

Anil S. Tavildar after retiring as a Principal, he was working as Professor Emeritus at Vishwakarma Institute of Information Technology, Pune, India. He obtained his B.E. from University of Pune and PhD (Communication Engineering) from I.IT. Delhi. He has total of 43 years of experience (28 years in industry and 15 years in teaching). His research interests include signal/ image processing, wireless communications and biometrics. Professor Tavildar is a Senior Member, IEEE USA, Fellow Member of IETE, India and Founder Member of ICIET, India. 


\section{Publisher's Note}

Springer Nature remains neutral with regard to jurisdictional claims in published maps and institutional affiliations.

\section{Author details}

'Department of E\&TC Engineering, Vishwakarma Institute of Information Technology, SPPU, Pune, India. 'Department of E\&TC Engineering, MKSSS's Cummins College of Engineering, SPPU, Pune, India.

Received: 8 January 2019 Accepted: 30 April 2019

Published online: 28 May 2019

\section{References}

1. P. Wild, H. Hofbauer, J. Ferryman, A. Uhl, Quality-based iris segmentationlevel fusion. EURASIP J. Inf. Secur. 2016, 25 (2016). https://doi.org/10.1186/ s13635-016-0048-X

2. U. Gawande, Y. Golhar, Biometric security system: a rigorous review of unimodal and multimodal biometrics techniques. Int. J. of Biometrics 10(2), 142-175 (2018).

3. S. Bharadwaj, M. Vatsa, R. Singh, Biometric quality: a review of fingerprint, iris and face. EURASIP J. Image Video Proc. 1, 1-28 (2014).

4. A. Ross, K. Nandkumar, A. Jain, Handbook of Multi biometrics Vol. 6, (Springer-Verlag US, 2006).

5. K. Kumar, M. Farik, A review of multimodal biometric authentication systems. Int. J. Sci. Technol. Res. 5(12), 5-9 (2016).

6. Gopal, S. Srivastava, S. Bhardwaj, S. Bhargava, Fusion of palm-phalanges print with palm print and dorsal hand vein. Appl. Soft Comput. 47, 12-20 (2016).

7. S. Baghel, T.K. Sahu, K. Varma, Multimodal biometric system advantages over unimodal biometric system authentication technology. Int. J. Innov. Sci., Eng. Technol. 4(12), 204-207 (2017).

8. G. Chaudhary, S. Srivastava, Accurate human recognition by score-level and feature-level fusion using palm-phalanges print. Arab. J. Sci. Eng. 18 43(2), 543-554 (2017).

9. A. Bansal, S. Arora, S. Singh, A survey on fingerprint recognition system. Int. J. Comput. Sci. Eng. 6(5), 1192-1197 (2018).

10. M. Kaur, S.S. Khurmi, Biometric recognition system: a review. Int. J. Comput. Sci. Eng. 5(9), 40-45 (2017).

11. P.D. Deshpande, A.S. Tavildar, Y.H. Dandwate, E. Shah, Fusion of dorsal palm vein and palm print modalities for higher security applications. Conf. Adv. Sig. Proc. (CASP), 201-206 (2016). https://doi.org/10.1109/CASP.2016. 7746165.

12. A. Joshi, P. Deshpande, A.S. Tavildar, Enhancing accuracy for personal identification using hierarchical based fusion of finger geometry and palm print modalities. Int. Conf. Electr. Commun. Syst., 111-116 (2014). https://doi. org/10.1109/ECS.2014.6892663.

13. H. Imtiaz, S.A. Fattah, A wavelet-based dominant feature extraction algorithm for palm-print recognition, science direct. Digital Signal Process. 23, 244-258 (2013).

14. E.W.K. Yih, G. Sainarayanan, A. Chekima, G. Narendra, Palm Print Identification Using Sequential Modified Haar Wavelet Energy (IEEEInternational Conference on Signal processing, Communications and Networking, Chennai, 2008), pp. 411-416.

15. M.P. Dale, M.A. Joshi, N. Gilda, in IEEE Seventh International Conference on Advances in Pattern Recognition. Texture based palmprint identification using DCT features (2009), pp. 221-224.

16. Z. Guo, D. Zhang, L. Zhang, Empirical study of light source selection for palm print recognition. Pattern Recogn. 32, 120-126 (2011).

17. G.K.O. Michael, T. Connie, A.B.J. Teoh, A contactless biometric system using multiple hand features, science direct. J. Vis. Commun. Image R 23, 10681084 (2012)

18. K.-S. Wu, J.-C. Lee, T.-M. Lo, A secure palm vein recognition system. J. Syst. Softw. 86, 2870-2876 (2013).

19. S. Sasidharan, M. Azoth, Study on palm vein authentication. Int. J. Comput. Sci. Eng. 3(1), 28-32 (2015).

20. B. Raghavendra, Texture based features for robust palm print recognition: a comparative study. EURASIP J. Inf. Secur. (20152015:5). https://doi.org/10. 1186/s13635-015-0022-z.

21. S. Khellat-Kihela, R. Abrishambaf, J.L. Monteiro, M. Benyettou, Multimodal fusion of the finger vein, fingerprint and the finger-knuckle-print using kernel fisher analysis. Appl. Soft Comput. 42, 439-447 (2016).
22. L. Rupali, P.D. Deshmukh, G.Y. P.C.C.S, A.M.N. Siddiqui, Combination approach to score level fusion for multimodal biometric system by using face and fingerprint (IEEE International Conference on Recent Advances and Innovations in Engineering, Jaipur, 2014). https://doi.org/10.1109/ICRAIE. 2014.6909320.

23. K. Vishi, V. Mavroeidis, An evaluation of score level fusion approaches for fingerprint and finger-vein biometrics (Proceedings of the 10th Norwegian Information Security Conference, Oslo, 2017).

24. H.L. Van, Detection, estimation, and modulation theory (Wiley- US, 2004), ISBN: 978-0-471-46382-5.

25. M. Barkat, Signal detection and estimation, Artech house. Technol. Eng., Boston \& London (2005). p. 714, ISBN: 9781580530705.

26. L. Yi-bo, L. Jun-jun, Harris corner detection algorithm based on improved Contourlet transform. Adv. Contr. Eng. Inform. Sci. Proc. Eng. 15, 2239-2243 (2011).

27. G. Seshikala, D.U. Kulkarni, M.N. Giriprasad, Palm print feature extraction using multi scale wavelet edge detection method. Int. J. Adv. Res. Elec. Electr. Instrum. Eng.ISSN: 2278- 8875 1, 24-28 (2012).

\section{Submit your manuscript to a SpringerOpen ${ }^{\circ}$ journal and benefit from:}

- Convenient online submission

- Rigorous peer review

- Open access: articles freely available online

- High visibility within the field

- Retaining the copyright to your article

Submit your next manuscript at $\boldsymbol{\nabla}$ springeropen.com 